\title{
Determining Design Characteristics of Automobile Seats Based On Fuzzy Axiomatic Design Principles
}

\author{
Selcuk CEBI* \\ Department of Industrial Engineering, Karadeniz Technical University, \\ 61080, Trabzon, Turkey \\ Cengiz KAHRAMAN \\ Department of Industrial Engineering, Istanbul Technical University, \\ Macka 34367, Istanbul, Turkey \\ kahramanc@itu.edu.tr \\ www.itu.edu.tr \\ Received: 07-07-2009 \\ Accepted: 08-12-2009
}

\begin{abstract}
The performance of a product depends on if it is created in terms of expected functional requirements. A product is designed to satisfy some certain tasks. In this study, the design of an automobile seat is handled. Automobile seat design procedure is based on experience and trial-and-error method, rather than structured and scientific methods. Axiomatic design is a design theory providing a systematic approach to engineering design. We propose a new product development algorithm based on axiomatic design principles. The independence axiom of axiomatic design is extended to fuzzy environment. Then, an automobile seat design process is taken into consideration by the proposed algorithm. The design characteristics of an automobile seat and their priorities are determined. Moreover, the design dimensions of an automobile seat are defined for Turkish drivers.
\end{abstract}

Keywords: Automobile seat design, axiomatic design, independence axiom, fuzzy logic, analytic hierarchy process.

\section{Introduction}

Automobiles are the indispensable item of our daily life. Automobile manufacturers want to well ahead to their competitors by presenting issues regarding to comfort, safety, and the best quality. As a way of meeting customers' increased need and expectation of vehicle comfort, car makers have been seeking more effective ways to improve automobile seats. A comfortable seat has an important role in the perception of an automobile's overall comfort and quality. Consumers' expectations for an automobile seat comfort continue rising because the role of the automobiles in our life has been increasing day by day. An automobile seat represents a work environment which must optimally position the occupant to perform the driving task to meet various safety requirements, and to be acceptable in terms of the driver's expectations. ${ }^{1}$ Hence, the design of automobile seats must be distinguished from the design of office chairs.

An automobile seat consists of a headrest, a backrest, and a cushion and it is built in three parts: a metal armature, foam injected in a matrix, and a dress which covers the foam and armature. These parts are connected to each other by three joints: one for the connection between seat cushion and its surroundings, one for the connection between seat cushion and seat back, and one for the connection between seat back and headrest. These joints allow adjustment in the seat back angle and head restraint angle. ${ }^{2}$ An automobile seat is created by a combination of seat types (i.e. full bench, split bench, and bucket), content (manual or power adjuster, manual or power recliner, adjustable or fixed head restraint, etc.), features (lumbar, front and/or rear

\footnotetext{
*Corresponding author. Tel.: +90 212293 1300-2746; Fax: +90 2122407260

E-mail address: cebiselcuk@gmail.com (S. Cebi)
} 
cushion tilt, seat heaters, etc.), trim styles (i.e. base level, mid level, and up level), and fabrics (i.e. cloth, vinyl, leather). ${ }^{3}$

In the literature, there are a great number of publications based on automobile seat design or seat comfort. ${ }^{4-15}$ In the literature, there are two common features of the studies: i) occupant anthropometry, seat geometry, and amount of the sitting time are the most citied factors that affect automobile seat comfort, ii) a subjective evaluation procedure is commonly used to define comfort characteristics. Since the seat design depends on the customer preferences automobile seats are developed in an iterative manner. The automobile seat design is created via subjective feedback which is obtained by a questionnaire. ${ }^{8}$ In a subjective evaluation, judgments of the participants, which are related a product are collected and they are evaluated by using some features of the product and the agreement level with respect to the objective.

The current automobile seat design process needs to be changed since it is fitfully successful. In this context, there has been a growing recognition of the need for automobile seat researchers to establish a theoretical and methodological foundation. ${ }^{3}$ In this study, the independence axiom which is the first axiom of the axiomatic design principles is used for the conceptual design of an automobile seat. The main goal of the proposed study is to determine the most important characteristics of an automobile seat design and present design specifications. Axiomatic Design (AD) is proposed by $\mathrm{Suh}^{16}$ to establish a scientific basis for the improvement of design activities by providing designers with a theoretical foundation based on logical and rational thought process and tools. AD is founded to provide a thinking process to create a new design and/or to improve the existing design. ${ }^{16}$ Axiomatic design methodology has been used to represent a variety of design problems since the date it was proposed. The applications of axiomatic design principles can be classified into four main domains such as product design, system design, manufacturing system design, and software design. Moreover, the number of the papers which are related to axiomatic design applications in decision making area has been increasing tremendously in the last few years.

The rest of this paper is organized as follows; Section 2 introduces the fundamentals of AD theory. The proposed methodology is given in Section 3. Section 4 includes an application of an automobile seat design based on occupant comfort. Finally, section 5 presents the concluding remarks.

\section{Design Axioms}

AD method proposes a scientific and systematic basis providing a structure to design processes for engineers. $\mathrm{AD}$ makes designers more creative, reduces the random search process, minimizes the iterative trial-and-error process, and determines the best designs among the proposed designs. The essence of the axiomatic design involves a continuous interplay between what customers want and how their needs are achieved. ${ }^{16-17}$ The AD theory consists of four domains: (1) Customer Domain, (2) Functional Domain, (3) Physical Domain, and (4) Process Domain. Each domain is characterized by a set of information: Customer Attributes (CAs) in the Customer Domain; Functional Requirements (FRs) and constraints in the Functional Domain; Design Parameters (DPs) in the Physical Domain; and Process Variables (PVs) in the Process Domain. These domains are linked through several mappings as shown in Figure 1. There are three types of mappings; (1) Customer Domain- Functional Domain, (2) Functional Domain Physical Domain where possible design parameters are determined for each function, and (3) Physical Domain - Process Domain. The domain on the left relative to the domain on the right represents "what customers/designers want to achieve"; the domain on the right represents the design solution of "how customers'/designers' requirements defined in the left domain are satisfied". ${ }^{17}$

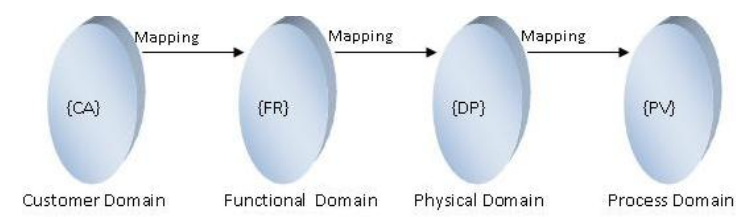

Figure 1. Design mappings and domains

The AD method continues from the high level of abstraction to the detailed modularity elements by zigzagging between domains to decompose the design problem (Figure 2). ${ }^{17}$ 




Figure 2. Decomposition by zigzagging

AD theory is based on two axioms, independence axiom and information axiom. Formal definitions of axioms are as follows ${ }^{16}$ :

Axiom 1. The Independence Axiom: Maintain the independence of the functional requirements

Axiom 2. The Information Axiom: Minimize the information content of the design.

The first axiom, the independence axiom, is about maintaining the independency between functional requirements (FRs) and design parameters (DPs). In other words, the design can be acceptable if and only if FRs must be satisfied by DPs without affecting any other FR. FRs are defined as the minimum set of independent requirements that characterizes design goals. ${ }^{16-17}$ The relationship between FRs and DPs is defined by Equations (1-3).

$\{F R\}=[R]\{D P\}, \quad R=\left\lfloor a_{i j}\right\rfloor_{m x n}$

$R=\left[\begin{array}{cccc}a_{11} & a_{12} & \ldots & a_{1 n} \\ a_{21} & a_{22} & \ldots & a_{2 n} \\ \ldots & \ldots & \ldots & \ldots \\ a_{m 1} & a_{m 2} & a_{m 3} & a_{m n}\end{array}\right]$

$a_{i j}=\frac{\partial F R_{i}}{\partial D P_{j}}$

where $R$ is a relation matrix between $F R$ and $D P$. The relation matrix consists of " 1 " and " 0 " elements " 1 " represents a relation between any FR and any DP while " 0 " represents no relation. In $\mathrm{AD}$ methodology, there are three types of design with respect to the number of FRs and DPs; (1) if the number of DPs is larger than the number of FRs, the design is named as coupled. (2) If the number of FRs is larger than the number of DPs, the design is named as redundant, (3) if the number of DPs is equal to the number of FRs, the design can be named coupled, decoupled, or uncoupled. If the number of DPs is equal to the number of FRs, the types of design mentioned above are defined according to the relationships between FRs and DPs. If the relation matrix is diagonal, the design is uncoupled (Eq. 4). This type of design is the ideal design. If the design matrix is lower triangular, the design is decoupled (Eq. 5). Otherwise, the design is coupled. ${ }^{15}$

$$
\begin{aligned}
A & =\left[\begin{array}{cccc}
a_{11} & 0 & \ldots & 0 \\
0 & a_{22} & \ldots & 0 \\
\ldots & \ldots & \ldots & \ldots \\
0 & 0 & 0 & a_{m n}
\end{array}\right] \\
A & =\left[\begin{array}{cccc}
a_{11} & 0 & \ldots & 0 \\
a_{21} & a_{22} & \ldots & 0 \\
\ldots & \ldots & \ldots & \ldots \\
a_{m 1} & a_{m 2} & a_{m 3} & a_{m n}
\end{array}\right]
\end{aligned}
$$

The second axiom, information axiom, is about minimizing the information content of the design. In other words, among all proposed solutions that satisfy the independence axiom, the best design is the design that has the minimum information content. Information axiom provides a conventional method to assess the designs to select the best one. The selection process is based on the criterion which states that the design with the highest probability of FR success is the best design. ${ }^{17}$ If the probability of success for a given FR is $\mathrm{p}$, the information content is calculated by Eq. (6);

$$
I_{i}=\log _{2} \frac{1}{p_{i}}
$$

If there are two or more FRs, the total information content is calculated as follows; ${ }^{16-17}$

$$
\begin{aligned}
& I_{\text {system }}=-\log _{2} P_{(m)} \\
& I_{\text {system }}=-\log _{2}\left(\prod_{i=1}^{m} P_{i}\right) \\
& I_{\text {system }}=-\sum_{i=1}^{m} \log _{2} P_{i}=\sum_{i=1}^{m} \log _{2}\left(1 / P_{i}\right)
\end{aligned}
$$


In Figure 3, system design, design range and system probability density function, and common range are illustrated.

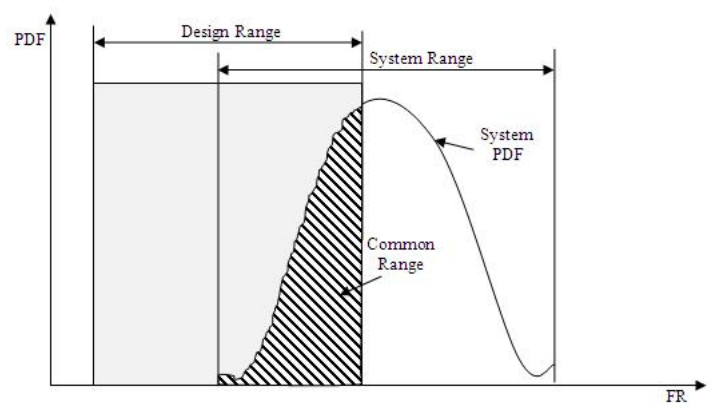

Figure 3. System design, design range, and common area

\section{Proposed Methodology}

The proposed methodology is based on the first axiom of the axiomatic design. The framework of the proposed methodology is given in Figure 4.

Step 1: Determine design groups. While determining the design group, the potential user population of the designed product must be taken into consideration.

Step 2: Define FRs in the functional domain. In this step, to define design parameters, functional requirements are determined to satisfy customer needs. To determine FRs, customer surveyors, interview, and literature review are utilized.

Step 3: Define DPs in the physical domain. Design parameters are defined to satisfy the defined FRs.

Step 4: Decompose FRs and DPs. FRs and DPs at the top level are decomposed until to obtain applicable design parameters.

Step 5: Construct the design matrix and evaluate the relations between FRs and DPs. In this study, the evaluation of relations is realized by using a linguistic scale with fuzzy membership functions making our method different from the conventional axiomatic design methodology. In the axiomatic design methodology, relations are defined by 0 or 1 . If there is a relation between a FR and a DP, it is depicted by 1 in the relation matrix. Otherwise, the relation is denoted by 0 as it is mentioned before. However, in real case problems, sometimes, the relations between FRs and DPs can be unknown or uncertain. Moreover, there can be a little or indirect relationship between a FR and a
DP such that this relation can be negligible. Hence, the conventional axiomatic design principles can become insufficient to define the degrees of relations between FRs and DPs under uncertainty or fuzziness. When the relationships between FRs and DPs are unknown or when they are weak relationships, these relationships can be defined by linguistic descriptions. Linguistic descriptions are the formal representation of systems which are made through the fuzzy set theory, fuzzy relations, and fuzzy operators. Linguistic scales describe the evaluation system of human logic which is used in daily life. ${ }^{18}$ Expressing preferences in a qualitative way by using linguistic terms is better than it is in a quantitative way by using precise numbers. ${ }^{19}$ In this study, the linguistic scale shown in Figure 5 is used to describe the relations between FRs and DPs when a relation cannot be assessed precisely. Seven linguistic terms are defined in an interval ranging from 0 to 1 shown in Figure 5. By the given linguistic scale, relations between FRs and DPs can be assessed by using the interval between $[0,1]$ instead of only 0 or 1 .

The evaluation of the relation matrix is implemented by a group of experts directly (Eq. 10).

$$
R^{k}=\left[\begin{array}{llll}
\widetilde{a}_{11} & \tilde{a}_{12} & \ldots & \widetilde{a}_{1 n} \\
\widetilde{a}_{21} & \widetilde{a}_{22} & \ldots & \widetilde{a}_{2 n} \\
\vdots & \vdots & \vdots & \vdots \\
\tilde{a}_{m 1} & \tilde{a}_{m 2} & \ldots & \tilde{a}_{m n}
\end{array}\right], \quad k=1,2, \ldots, K
$$

where $m$ and $n$ are the number of FRs and DPs, respectively; $k$ is the number of experts; $R^{k}$ is the fuzzy design matrix belonging to $k^{\text {th }}$ expert.

Step 6: Aggregate the experts' assessments. If the evaluation of a relation matrix is implemented by each expert separately, the arithmetic mean method is used for aggregation.

$\left\{F R_{i}\right\}=\left\{R^{c}\right\}\left\{D P_{j}\right\}$

$\left[R^{C}\right]=\left[a_{i j}\right], i=1,2,3, \ldots, m$ and $j=1,2,3, \ldots, n$

where $F R_{i}, D P_{j}$, and $R_{c}$ are $i^{\text {th }}$ functional requirement, $j^{\text {th }}$ design parameter, and fuzzy co-decision matrix that shows the relations between FRs and DPs, respectively. 


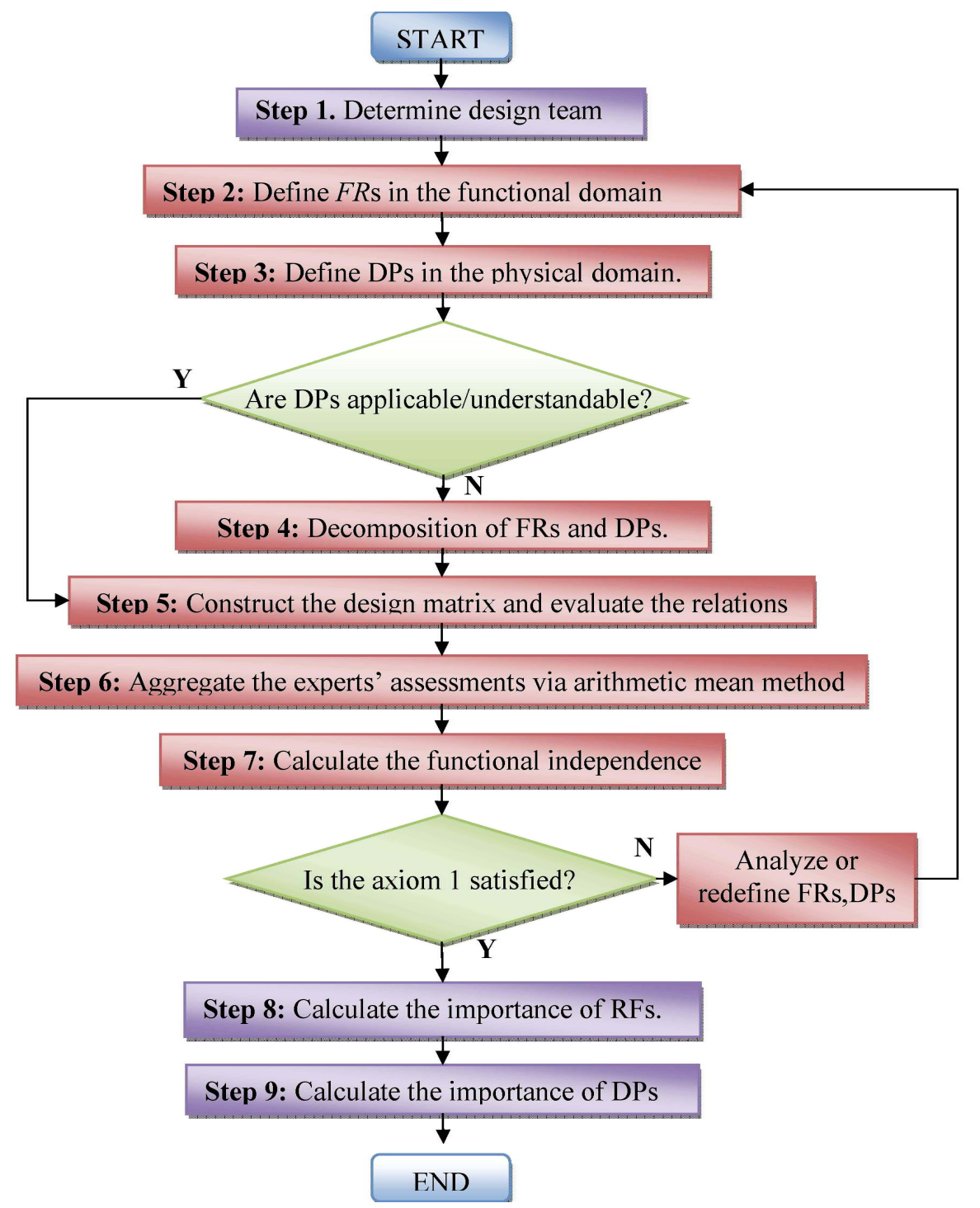

Figure 4. Framework of the proposed methodology

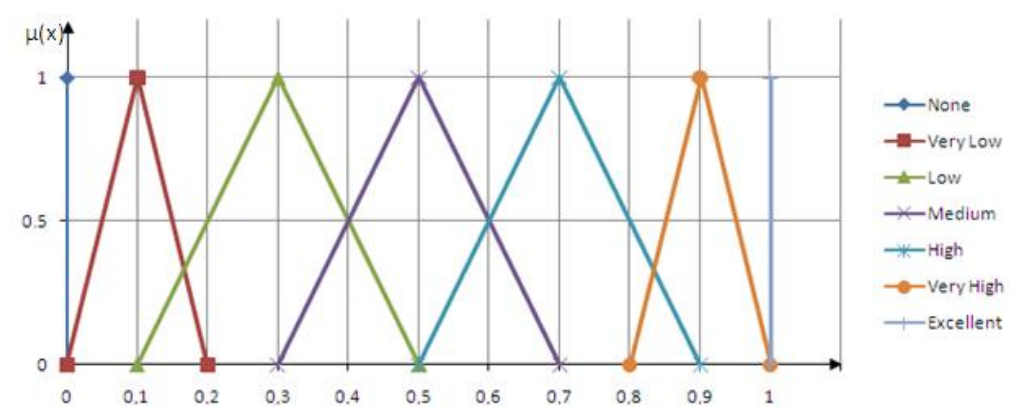

Figure 5. Linguistic scale for relationships 
Step 7: Calculate the functional independency. At first, the sequence of FRs is determined by Equations. 13 and 14 ;

$$
\begin{gathered}
S_{F R i}=\sum_{j=1}^{n} a_{i j}^{m} \\
S_{D P j}=\sum_{i=1}^{m} a_{i j}^{m}
\end{gathered}
$$

where $a_{i j}^{m}$ is the middle value of a fuzzy triangular number, which shows the relation between $F R_{i}$ and $D P_{j}$. $S_{F R i}$ and $S_{D P j}$ are the sequence scores of $F R_{i}$ and $D P_{j}$, respectively. FRs are ranked with respect to the sequence score in co-decision matrix from minimum to maximum considering the proposed design parameters. If there is any equality among the sequence scores of $F R$ s, the $F R$ which belongs to the biggest $S_{D P j}$ is written firstly. If both $S_{F R i}$ and $S_{D P j}$ of FRs are equal, the sequence can be made in the way whatever the designer wants. It must be noted that if there is a logical sequence among the DPs or FRs, this relation must be taken into consideration firstly. For example, $D P_{b}$ is created after $D P_{a} . D P_{b}$ is not written before $D P_{a}$ even if $S_{D P b}$ is bigger than $S_{D P a}$. Then Equation 15 is used to calculate the degree of functional independency.

$$
\widetilde{C}=\frac{\sum_{i=1}^{m} \sum_{j=i+1}^{m} \tilde{a}_{i j}}{\sum_{i=1}^{m} \sum_{j=i+1}^{m} 1}
$$

where $\widetilde{C}$ is the coupled ratio which denotes the degree of independence and $\tilde{a}_{i j}$ is the fuzzy relationship between the related $F R$ and $D P$. If $C=1$ or $C>\gamma$, the design is coupled and if $C=0$ or $C \leq \gamma$, the design is uncoupled or decoupled, where $\gamma$ is the value which shows the level of acceptable or tolerable relation defined by experts. The value larger than 0 or $\gamma$ indicates the coupled design (Eq.16).

If $C=0$ or $C \leq \gamma$ and

$$
\widetilde{c}=\frac{\sum_{i=1}^{m} \sum_{j=1}^{i-1} \tilde{a}_{i j}}{\sum_{i=1}^{m} \sum_{j=1}^{i-1} 1}=0,
$$

a design is uncouple. Otherwise it is decoupled. Here $\widetilde{c}$ is the uncoupled ratio.

Step 8: Calculate the importance of functional requirements. The importance of the functional requirements is calculated by pairwise comparisons. Buckley's method is used to obtain weights ${ }^{20-21}$ : The pairwise comparison matrix is given by Eq. (17) for any expert.

$$
\widetilde{C}_{k}=\left|\begin{array}{cccc}
1 & \widetilde{c}_{12} & \ldots & \widetilde{c}_{1 n} \\
\widetilde{c}_{21} & 1 & \ldots & \widetilde{c}_{2 n} \\
\vdots & \vdots & \vdots \vdots & \vdots \\
\widetilde{c}_{n 1} & \widetilde{c}_{n 2} & \ldots & 1
\end{array}\right|, k=1,2,3, \ldots . K
$$

where $\tilde{C}_{k}$ is a pairwise comparison matrix which belongs to $k^{\text {th }}$ expert for $F R_{m}$. The triangular fuzzy numbers are given by Eq.(18) for pairwise comparison matrices.

$$
\widetilde{c}_{i j}=\left\{\begin{array}{l}
(1,1,3),(1,3,5),(3,5,7),(5,7,9),(7,9,9), \\
\quad \text { if } i \text { is moreimportanthan } j \\
(1,1,1), \\
\quad \text { if } i \text { and } j \text { havethesameimportance } \\
(1,1,3)^{-1},(1,3,5)^{-1},(3,5,7)^{-1},(5,7,9)^{-1},(7,9,9)^{-1} \\
\quad \text { if } i \text { is lessimportanthan } j
\end{array}\right.
$$

The linguistic scale for triangular fuzzy numbers in Eq. (18) is given in Table 1.

Table 1. Linguistic scale for the weight matrix $^{21}$

\begin{tabular}{clc}
\hline Linguistic scales & Scale of fuzzy number & \\
\hline$(1,1,3)$ & Equally important & $(\mathrm{Eq})$ \\
$(1,3,5)$ & Weakly important & $(\mathrm{Wk})$ \\
$(3,5,7)$ & Essentially important & $(\mathrm{Es})$ \\
$(5,7,9)$ & Very strongly important & $(\mathrm{Vs})$ \\
$(7,9,9)$ & Absolutely important & $(\mathrm{Ab})$ \\
\hline
\end{tabular}

Then, the fuzzy weighted design matrix is calculated by Buckley's Method as follows:

$$
\begin{aligned}
& \tilde{r}_{i}=\left(\widetilde{c}_{i 1} \otimes \widetilde{c}_{i 2} \otimes \ldots \otimes \widetilde{c}_{i n}\right)^{1 / n} \\
& \tilde{w}_{F R i}=\tilde{r}_{i} \otimes\left(\tilde{r}_{1}+\widetilde{r}_{2}+\ldots+\widetilde{r}_{n}\right)^{-1}
\end{aligned}
$$

where $\widetilde{c}_{i n}$ is the fuzzy comparison value of $D P_{i}$ to $D P_{n}$ under the relevant $F R$, and $\widetilde{r}_{i}$ is the geometric mean of fuzzy comparison values of $D P_{i}$ to each $D P$ under $F R_{n}$. $\widetilde{w}_{F R i}$ is the fuzzy weight for $F R_{i}$. When there are more 
than one expert, geometric mean method is used to aggregate the experts' preferences before applying Eqs. 19-20.

Step 9: Calculate the importance of design parameters. The importance of design parameters is calculated in order to define most important parameters in the design. Sometimes, all functional requirements cannot be fully satisfied. Hence, the most important item that satisfies the functional requirements at most is determined in order to give it the design priority. Eq. 21 is used to calculate the weights of design parameters via a relation matrix.

$$
\widetilde{w}_{D P j}=\sum_{i=1}^{m} \widetilde{w}_{F R i} \otimes \widetilde{a}_{i j} \quad i=1,2, \ldots, m \text { and } j=1,2, \ldots n,
$$

where $\widetilde{w}_{D P}$ and $\widetilde{w}_{F R}$ are the fuzzy weights of design parameters and functional requirements, respectively. Then, the obtained fuzzy numbers are defuzzified into crisp values. Then, the normalization procedure is applied. Eqs. 22-23 are used to obtain crisp values for importance of functional requirements. Eq. 23 present both defuzzification and normalization procedures in one formula.

$$
\begin{aligned}
& T \widetilde{w}_{D P j}=w_{D P j}^{l}+w_{D P j}^{m}+w_{D P j}^{u} \\
& w_{D P j}=\frac{T \widetilde{w}_{D P j}}{\sum_{j=1}^{n} T \widetilde{w}_{D P j}}
\end{aligned}
$$

where $w_{D P j}^{l}, w_{D P j}^{m}$, and $w_{D P j}^{u}$ are the lower, middle, and upper values of triangular fuzzy numbers which depends on the importance of design parameters, respectively.

It is also possible to rank the design parameters by a fuzzy ranking approach without converting them to crisp numbers. Many ranking algorithms have been proposed in the literature. For instance, see a very recent approach to rank fuzzy numbers in ${ }^{22}$.

\section{Automobile Seat Design for Turkish Consumers}

In this section, characteristic features of an automobile seat are taken in to consideration based on the methodology given above.
Step 1. Determine the design team. The design team consists of a project manager, a product designer, and two mechanical engineers. The product designers and engineers are familiar with ergonomics and automotive seat design. The responsibility of the project manager is to lead this design process and supply financial support.

Step 2: Define FRs in the functional domain. To determine the customers' expectations a survey is performed. Eighteen volunteers of taxi drivers are selected as the participant group of the survey with respect to two criteria. The first one is that drivers must have at least five years taxi-driver experiences. The second criterion is that drivers must not have any musculosketal disorders. The main reason to select taxi drivers as an experimental set is that they more frequently spend longer hours in their vehicles by sitting. The overall expectations of the taxi drivers from an automobile seat are summarized in Table 2. The factors listed in Table 2 are the expectations of participants and they are related to seat characteristics. Some factors such as reachability (to glove box, to gearshift stick, to hand brake) and good visibility through side window are not taken in to consideration since they are not stated by the participant group.

\begin{tabular}{ll} 
Table 2. Drivers' & expectations from an automobile seat \\
\hline Main Factors & Sub Factors \\
\hline \multirow{4}{*}{ Good visibility } & Displays \\
& Instrument panel controls \\
& Controls on the left of steering wheel \\
& Through windshield \\
Reach & Control buttons \\
& Pedals \\
& Backrest adjustability control \\
& Height adjustability control \\
Adjustments & Fore-and-aft control \\
& Headrest adjustability control \\
& Backrest adjustability control \\
& Altitude adjustability control \\
& Knee-Buttock \\
& Back (Buttock-Neck) \\
& Neck-Head \\
Comfort & Head \\
& Arms \\
\hline
\end{tabular}

An automobile seat should satisfy drivers' comfort and facilitate to retain the control of a car. The drivers' comfort means that the components of a seat such as backrest, headrest, and cushion must be comfortable and adjustments of the seat must be made easily. The most important mission of a seat is that it should make easy 
to reach pedals and to satisfy good visibility to provide driving safety.

Step 3: Define DPs in the physical domain. Design parameters for an automobile seat are as follows; travel path, cushion width, cushion length, cushion stiffness, cushion bolsters, backrest height, backrest width, backrest stiffness, backrest bolsters, lumbar support, headrest length, and headrest width (Figure 6).

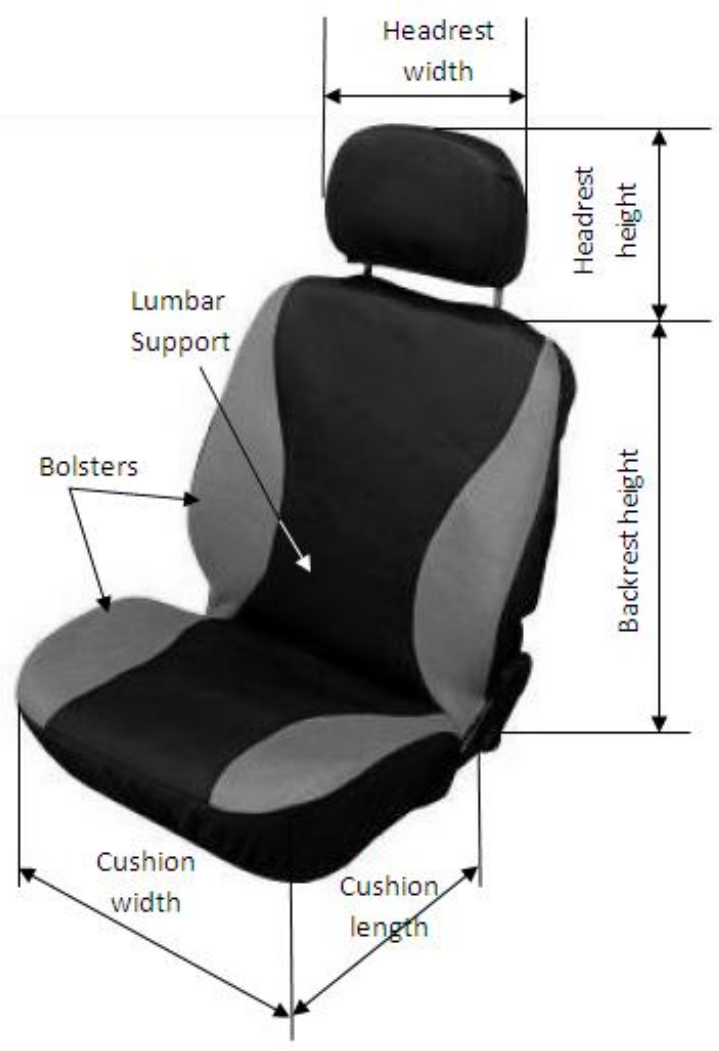

Figure 6. An automobile seat

In Figure 7, the travel path of a seat is given.

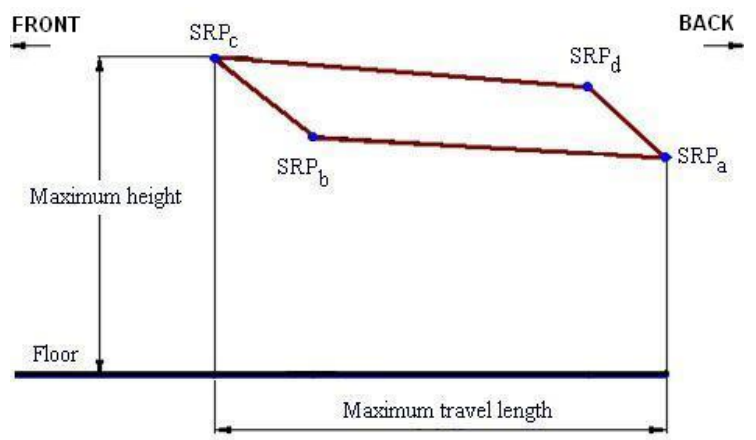

Figure 7. Seat travel path; seat reference point (SRP)
Step 4: Decompose of FRs and DPs. The hierarchy of the FRs and DPs defined in the previous steps is presented in Figure A1. For the seat design, the following functional requirements and design parameters are defined;

FR: Driver seat design that satisfy the customers' expectations

DP: Ergonomic seat design

FR1: Satisfy the good visibility

DP1: Determine the vertical travel range value of seat

FR2: Satisfy the easy reach to controls and pedals

DP2: Determine the horizontal travel range value of seat

FR3: Satisfy the adjustments easily

DP3: Determine the adjustment design parameters

FR31: Make the backrest adjustability control easy

DP31: Backrest adjustability control button

FR32: Make the altitude adjustability control easy

DP32: Adjustability altitude control button

FR33: Make the fore-and-aft control easy

DP33: Fore-and-aft control button

FR34: Make the head rest control easy

DP34: Headrest control button

FR4: Satisfy the drivers comfort

DP4: Determine the comfort design parameters

FR 41: Upper Leg comfort

DP41: Cushion length

FR42: Hip comfort

DP42: Cushion width

FR 43: Lumbar comfort

DP 43: Lumbar support

FR44: Middle back comfort

DP44: Seatback width

FR 45: Upper back comfort

DP45: Seat Height

FR46: Headrest comfort

DP46: Headrest height

Step 5: Construct the design matrix and evaluate the relations between FRs and DPs. The design team constructs the design matrix via brain storming. The expert opinions are received in linguistic form by using the scale given in Figure 5. The design equations are determined as follows:

$$
\left\{\begin{array}{l}
F R 1 \\
F R 2 \\
F R 3 \\
F R 4
\end{array}\right\}=A\left\{\begin{array}{l}
D P 1 \\
D P 2 \\
D P 3 \\
D P 4
\end{array}\right\}
$$




$$
\left\{\begin{array}{l}
F R 1 \\
F R 2 \\
F R 3 \\
F R 4
\end{array}\right\}=\left[\begin{array}{cccc}
E & V L & N & N \\
H & E & N & N \\
N & N & A_{3} & N \\
N & N & N & A_{4}
\end{array}\right]\left\{\begin{array}{l}
D P 1 \\
D P 2 \\
D P 3 \\
D P 4
\end{array}\right\}
$$

where $A_{3}$ and $A_{4}$ are the relation matrices between FR3-DP3 and FR4-DP4, respectively. So, the design equation for $F R 3-D P 3$ is given as follow;

$$
\begin{aligned}
& \left\{\begin{array}{l}
F R 31 \\
F R 32 \\
F R 33 \\
F R 34
\end{array}\right\}=A_{3}\left\{\begin{array}{l}
D P 31 \\
D P 32 \\
D P 33 \\
D P 34
\end{array}\right\} \\
& \left\{\begin{array}{l}
F R 31 \\
F R 32 \\
F R 33 \\
F R 34
\end{array}\right\}=\left[\begin{array}{llll}
E & N & N & N \\
N & E & N & N \\
N & N & E & N \\
N & N & N & E
\end{array}\right]\left\{\begin{array}{l}
D P 31 \\
D P 32 \\
D P 33 \\
D P 34
\end{array}\right\}
\end{aligned}
$$

According to the Eq. 27, the design is uncoupled design. It means that each control button must have only and only one function. For example, fore-and-aft position control button must be used to control horizontal travel of the seat. Hence, for the each adjustment, only one control button must be used. And also the location of control buttons must be in different areas on the seat such that their location must be suitable with human logic and body. Therefore, fore-and-aft control button must be located in front of the seat. Moreover, the dimension and shape of the control button must be wellmatched with an adult's hand shape.

The design equation for the FR4-DP4 is given as follow;

$\left\{\begin{array}{l}F R 41 \\ F R 42 \\ F R 43 \\ F R 44 \\ F R 45 \\ F R 46\end{array}\right\}=A_{4}\left\{\begin{array}{l}D P 41 \\ D P 42 \\ D P 43 \\ D P 44 \\ D P 45 \\ D P 46\end{array}\right\}$

$$
\left\{\begin{array}{l}
F R 41 \\
F R 42 \\
F R 43 \\
F R 44 \\
F R 45 \\
F R 46
\end{array}\right\}=\left[\begin{array}{cccccc}
E & H & N & N & N & N \\
M & E & L & N & N & N \\
N & L & E & H & V L & N \\
N & N & H & E & M & N \\
N & N & N & H & E & N \\
N & N & N & N & N & E
\end{array}\right]\left\{\begin{array}{l}
D P 41 \\
D P 42 \\
D P 43 \\
D P 44 \\
D P 45 \\
D P 46
\end{array}\right\}
$$

According to Eq. 29, the design is seemed to be a coupled design. Substituting Eq. 27 and Eq. 29 into Eq. 25, we get Eq. 30 .

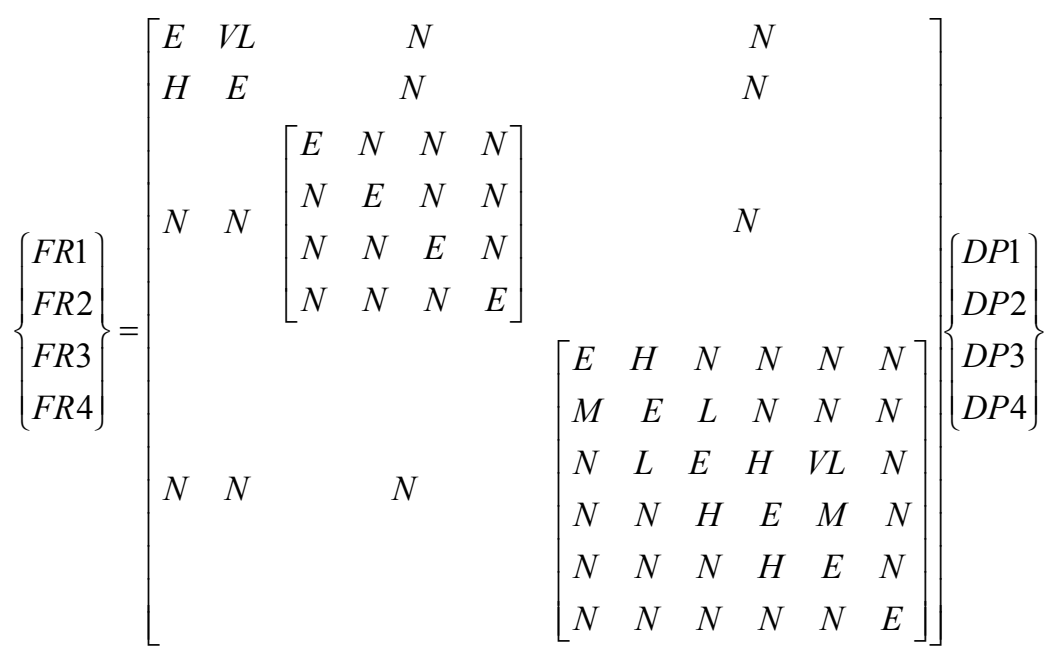

Step 6: Aggregate the experts' assessments. Since the relation matrix is obtained by brain storming, it does not need to calculate an arithmetic mean for the aggregation phase.
Step 7. Calculate the functional independency. In this step, substituting process of numerical values is started by sub-matrices. Then, the functional independency value of the relation matrix is obtained. So, the relation matrix $A_{4}$ is given as in Eq. 31 . 


$\begin{array}{|cccccc|}(1,1,1) & (0.8,0.9,1) & (0,0,0) & (0,0,0) & (0,0,0) & (0,0,0) \\ (0.3,0.5,0.7) & (1,1,1) & (0.1,0.2,0.5) & (0,0,0) & (0,0,0) & (0,0,0) \\ (0,0,0) & (0.1,0.3,0.5) & (1,1,1) & (0.8,0.9,1) & (0,0.1,0.2) & (0,0,0) \\ (0,0,0) & (0,0,0) & (0.8,0.9,1) & (1,1,1) & (0.3,0.5,0.7) & (0,0,0) \\ (0,0,0) & (0,0,0) & (1,1,1) & (0.8,0.9,1) & (1,1,1) & (0,0,0) \\ (0,0,0) & (0,0,0) & (0,0,0) & (0,0,0) & (0,0,0) & (1,1,1)\end{array}$

The sequence scores of $F R$ s are as follows; $S_{F R 41}=1.9$; $S_{F R 42}=1.7 ; S_{F R 43}=2.3 ; S_{F R 44}=2.4 ; \quad S_{F R 45}=2.9$; $S_{F R 46}=1$. Hence the new sequence of the $F R \mathrm{~s}$ is given in Eq. 32. The functional independency of the matrix is $\widetilde{C}=(0.09,0.12,0.18)$. According to Figure 5 , the obtained value is in the $V L$ range. So the coupling of the design is thought to be negligible.

$$
\left\{\begin{array}{l}
F R 46 \\
F R 42 \\
F R 41 \\
F R 43 \\
F R 44 \\
F R 45
\end{array}\right\}=A_{4}\left\{\begin{array}{l}
D P 46 \\
D P 42 \\
D P 41 \\
D P 43 \\
D P 44 \\
D P 45
\end{array}\right\}
$$

The functional independency value of the relation matrix $A_{3}$ is $\widetilde{C}=(0,0,0)$ since it is an uncoupled design. Substituting the obtained functional independency values in the Eq. 25, Eq. 33 is obtained.

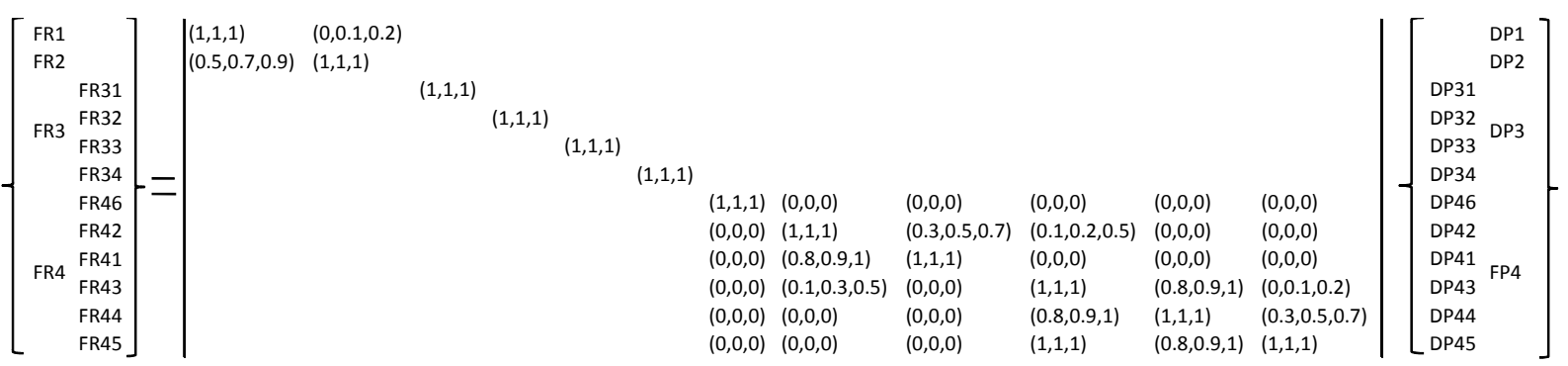

Hence, the functional independency value of the design is obtained as $\tilde{C}=(0.02,0.04,0.05)$. And also, this value is in the $V L$ range. Hence, the design is decoupled design and the uncoupled ratio of the design is $\tilde{c}=(0.06,0.07,0.08)$.

Step 8: Calculate the importances of functional requirements. The importances of the defined functional requirements are calculated by using Eqs. 17-20. The evaluation phase is implemented by the design team. The obtained weights are given in Table A1.

Step 9: Calculate the importances of design parameters. The importances of the design parameters given in Table 3 are calculated by using Eqs. 21 and 23 . According to the results, the most important design factor is the vertical travel range of a seat. The second is the horizontal travel range of a seat. Since the horizontal and vertical travel ranges affect the visibility and reachability to control buttons, respectively, they are important features for an automobile seat design. The adjustment controls have also the same importance. And lumbar support is the most important design factor in terms of drivers' comfort.

The calculated weight coefficients can be used to obtain the optimum design that satisfies the customers' expectations largely. Now, the dimensions of a seat are determined based on the anthropometric dimension of Turkish people. Some anthropometric dimensions belonging to Turkish people are given in Table $4 .{ }^{23}$ 
Table 3. The importances of design parameters

\begin{tabular}{lllc}
\hline & & Fuzzy Weights & Normalized Crisp Weights \\
\hline DP1 & & $(0.665,1.258,2.599)$ & 0.459 \\
DP2 & & $(0.37,0.814,1.266)$ & 0.249 \\
& DP31 & $(0.0099,0.0198,0.0792)$ & 0.011 \\
DP3 & DP32 & $(0.0099,0.0198,0.0792)$ & 0.011 \\
& DP33 & $(0.0099,0.0198,0.0792)$ & 0.011 \\
& DP34 & $(0.0099,0.0198,0.0792)$ & 0.011 \\
& DP41 & $(0.00612,0.0376,0.18036)$ & 0.023 \\
& DP42 & $(0.01053,0.06704,0.3708)$ & 0.045 \\
DP4 & DP43 & $(0.02556,0.11792,0.6066)$ & 0.076 \\
& DP44 & $(0.02196,0.1064,0.5472)$ & 0.069 \\
& DP45 & $(0.00702,0.04688,0.26388)$ & 0.032 \\
DP46 & $(0.0018,0.0048,0.0252)$ & 0.003 \\
\hline
\end{tabular}

Table 4. Anthropometric dimensions for Turkish adults

\begin{tabular}{lllllllll}
\hline Dimension & \multicolumn{4}{c}{ Men } & \multicolumn{4}{c}{ Women } \\
& 5th & 50th & 95th & STD & 5th & 50th & 95th & STD \\
\hline Buttock-popliteal length & 37.0 & 44.2 & 51.3 & 4.3 & 41.5 & 47.5 & 53.4 & 3.6 \\
Shoulder breadth & 37.0 & 47.5 & 57.9 & 6.4 & 32.7 & 36.6 & 40.5 & 2.4 \\
Hip breadth & 28.9 & 33.3 & 37.8 & 2.7 & 24.5 & 30.8 & 37.1 & 3.8 \\
Shoulder height & 129.3 & 141.6 & 153.9 & 7.5 & 119.0 & 130.7 & 142.4 & 7.1 \\
Hip height & 80.5 & 90.0 & 99.5 & 5.8 & 73.0 & 81.5 & 90.0 & 5.2 \\
Head length & 16.5 & 18.8 & 21.1 & 1.4 & 16.5 & 17.8 & 19.2 & 0.8 \\
Head breadth & 11.6 & 15.2 & 18.7 & 2.2 & 13.9 & 15.3 & 16.7 & 0.8 \\
Sitting height & 81.6 & 89.2 & 96.8 & 4.6 & 78.1 & 84.8 & 91.6 & 4.1 \\
\hline
\end{tabular}

Cushion length (DP41) is an important determinant of thigh support. If a cushion length is too long, it applies pressure on the posterior portion of the occupant's legs near the knee. Pressure in this area will lead to local discomfort and restricted blood flow to the legs. ${ }^{21}$ Therefore, cushion length is constrained by the buttock to popliteal length of the $5^{\text {th }}$ percentile female segment of the population. Hence, the cushion length should be at most $41.5 \mathrm{~cm}$. The design parameter cushion width (DP42) affects cushion lateral support. Therefore, cushion width should satisfy hip breadth of $95^{\text {th }}$ percentile female segment. For Turkish drivers, an automobile cushion should be at least $45 \mathrm{~cm}$ since the anthropometric measurement does not include a margin for clothing although it is given as $37 \mathrm{~cm}$ in Table 4 . The design parameter lumbar support (DP43) should be positioned between $105 \mathrm{~mm}$ and $150 \mathrm{~mm}$ from H-Point. ${ }^{21}$ In the upper seatback, the minimum width should support the chest breadth of a large male when reclining. Therefore, the seatback width and seat back height (DP44, DP45) should be at least $58 \mathrm{~cm}$ and 55 $\mathrm{cm}$, respectively. The headrest is a component that protects the neck when the automobile gets hit from behind. Hence, the design of headrest (DP46) is important in terms of driver safety. The headrest should be adjustable and its travel path should be $4 \mathrm{~cm}$. This travel path supports the $5^{\text {th }}$ percentile female segment and $95^{\text {th }}$ percentile male segment. The width of the headrest should be at least $18 \mathrm{~cm}$.

\section{Conclusion}

In this study, a design methodology is proposed based on the axiomatic design principles. The proposed methodology is applied to an automobile seat design. The methodology extends the independence axiom to fuzzy environment. In real case design problems, all factors that affect the design can not be known or predict precisely. And also there are complex relations among the design parameters has been determined as in seat design problem. According to the results, the most important feature of an automobile seat is travel range feature. As comfort requirements, the rank of the design parameters as follows; lumbar support, seatback width, cushion width, seatback height, cushion length, and headrest.

The main contributions of the proposed methodology to the literature are explained as follows:

$>$ The proposed methodology takes into consideration the unknown, unpredicted or weak relations between functional requirements and design parameters. 
$>$ The proposed methodology can be used to select the best design by using functional independence coefficient of the design. If there are two designs that consist of different design parameters and functional requirements, the best design is selected easily by the proposed algorithm

$>$ The importances of the design parameters are put forward by the proposed methodology. Hence, to obtain the optimum design, the importance of the design parameters determined in perspective of users help the designers.

For the further research, the proposed methodology can be combined by an optimization algorithm to determine the dimensions of any product.

\section{References}

1. D.R. Smith, D.M. Andrews, P.T. Wawrow, Development and evaluation of the Automotive Seating Discomfort Questionnaire (ASDQ), International Journal of Industrial Ergonomics 36 (2006) 141-149

2. M. M. Verver, R. de Lange, J. van Hoof, J. S. H. M Wismans, Aspects of seat modeling for seating comfort analysis, Applied Ergonomics 36(1) (2005) 33-42.

3. M. Kolich, A conceptual framework proposed to formalize the scientific investigation of automobile seat comfort, Applied Ergonomics 39(1) (2008) 15-27.

4. J. E. Brooks and K. C. Parsons, An ergonomics investigation into human thermal comfort using an automobile seat heated with encapsulated carbonized fabric (ECF), Ergonomics 42(5) (1999) 661-673.

5. H. G. Kim, G. H. Choe, B. H. Lee, Y. T. Cho, Structural analysis for lightweight design of a seat frame in automotive vehicles, Advances in Engineering Plasticity, 177-180 (2000) 799-804.

6. K. Ebe and M. J. Griffin Factors affecting static seat cushion comfort, Ergonomics 44(10) (2001) 901-921.

7. M. Kolich, Automobile seat comfort: occupant preferences vs. anthropometric accommodation. Applied Ergonomics 34(2) (2003) 177-184.

8. M. Kolich, Predicting automobile seat comfort using a neural network, International Journal of Industrial Ergonomics 33(4) (2004) 285-293.

9. M. Kolich, and S. M. Taboun, Ergonomics modeling and evaluation of automobile seat comfort, Ergonomics 47(8) (2004) 841-863.

10. G. Kyung and M. A. Nussbaum, Driver sitting comfort and discomfort (part II): Relationships with and prediction from interface pressure, International Journal of Industrial Ergonomics 38(5-6) (2008) 526-538.
11. G. Kyung, M. A. Nussbaum, K. Babski-Reeves, Driver sitting comfort and discomfort (part I): Use of subjective ratings in discriminating car seats and correspondence among ratings, International Journal of Industrial Ergonomics 38(5-6) (2008): 516-525.

12. T. G. Cengiz, and F. C. Babalik, The effects of ramie blended car seat covers on thermal comfort during road trials, International Journal of Industrial Ergonomics 39(2) (2009) 287-294.

13. M. Kolich, N. Seal, S. Taboun. Automobile seat comfort prediction: statistical model vs. artificial neural network, Applied Ergonomics 35(3) (2004) 275-284.

14. M. Grujicic, B. Pandurangan, G. Arakere, W.C. Bell, T. $\mathrm{He}, \mathrm{X}$. Xie, Seat-cushion and soft-tissue material modeling and a finite element investigation of the seating comfort for passenger-vehicle occupants, Materials \& Design, 30(10) (2009) 4273-4285

15. S. Bae, J. M.Lee, C. N Chu, Axiomatic design of automotive suspension systems, Cirp AnnalsManufacturing Technology 51(1) (2002) 115-118.

16. N.P. Suh, The Principles Of Design, (Oxford University Press, New York, 1990).

17. N.P. Suh, Axiomatic Design: Advances and Applications, (Oxford University Press, New York, 2001).

18. J. Lu, G. Zhang, D. Ruan, and F. Wu, Multi-Objective Group Decision Making Methods, Software and Applications with Fuzzy Set Techniques, (Imperial Collage Press, Singapore, 2007).

19. L. Martinez, M. J. Barranco, L. G. Perez, M. Espinilla, A Knowledge Based Recommender System with Multigranular Linguistic Information, International Journal of Computational Intelligence Systems, 1(3) (2008) 225 - 236

20. Chen, S.J., and Hwang, C.L., Fuzzy Multiple Attribute Decision Making Methods and Applications, (SpringerVerlag, Berlin, 1992)

21. T. Y. Hsieh, S. T. Lu, G. T. Tzeng, Fuzzy MCDM approach for planning and design tenders selection in public office buildings. International Journal of Project Management, 22, (2004), 573-584.

22. C Kahraman, A. C. Tolga An Alternative Ranking Approach and Its Usage in Multi-Criteria DecisionMaking, International Journal of Computational Intelligence Systems, 2 (3)(2009), 219-235.

23. A Iseri, and N. Arslan, Estimated anthropometric measurements of Turkish adults and effects of age and geographical regions, International Journal of Industrial Ergonomics doi:10.1016/j.ergon.2009.02.007. 

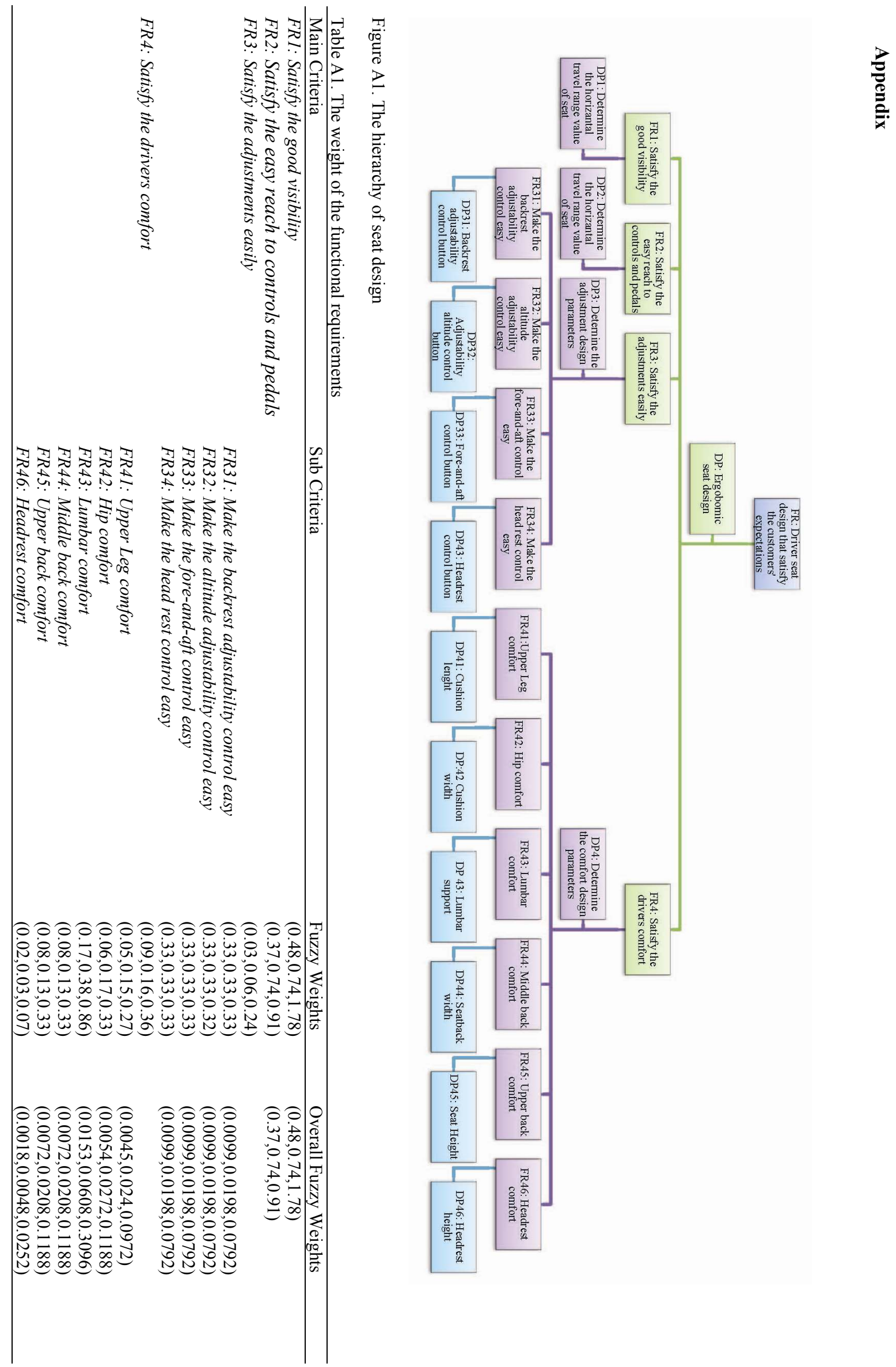